\title{
The Transmural Trauma Care Model (TTCM) for the rehabilitation of trauma patients is effective in improving patient related outcome measures: a non-randomized controlled trial
}

Suzanne H. Wiertsema ${ }^{1 *}$ (D) Johanna M. van Dongen², Edwin Geleijn ${ }^{3}$, Heleen Beckerman ${ }^{1}$, Frank W. Bloemers ${ }^{4}$, Raymond W. J. G. Ostelo ${ }^{5}$ and Vincent de Groot ${ }^{1}$

\begin{abstract}
Background: The Transmural Trauma Care Model (TTCM) is a refined post-clinical rehabilitation approach, in which a multidisciplinary hospital-based team guides a network of primary care physical therapists in the treatment of trauma patients. The objective of this study was to assess the effectiveness of the TTCM compared to regular care.

Methods: A controlled-before-and-after study was performed in a level 1 trauma center. The TTCM includes four elements: 1) a multidisciplinary team at the outpatient clinic, 2) coordination and individual goal setting for each patient by this team, 3) a network of primary care physical therapists, 4) E-health support for transmural communication. Intervention group patients were prospectively followed (3,6 and 9 months). The control group consisted of 4 clusters of patients who either had their first consultation at the outpatient clinic $0,3,6$ or 9 months ago. Outcomes included generic- and disease-specific health-related quality of life (HR-QOL), pain, functional status, patient satisfaction, and perceived recovery. Between-group comparisons were made using linear regression analyses. The recovery pattern of intervention group patients was identified using longitudinal data analysis methods.
\end{abstract}

Results: A total of 83 participants were included in the intervention group. In the control group, 202 participants were included (68 in the baseline cluster, 26 in the 3-month cluster, 51 in the 6-month cluster, 57 in the 9-month cluster). Between-group differences were statistically significant in favor of the intervention group for diseasespecific HR-QOL at 9 months, pain at 6 and 9 months, functional status at 6 and 9 months, patient satisfaction at 3, 6 and 9 months, and perceived recovery at 6 months. No significant differences were found between groups for generic HR-QOL at any time point. Generic HR-QOL, disease-specific HR-QOL, pain, and functional status significantly improved in a linear fashion among intervention group patients during the nine-month follow-up period.

Conclusions: This study provides preliminary evidence that the TTCM is effective in improving patient related outcome measures, such as disease-specific HR-QOL, pain and functional status. A multicenter, and ideally randomized controlled trial, is required to confirm these results.

(Continued on next page)

\footnotetext{
* Correspondence: s.wiertsema@amsterdamumc.nl

'Department of Rehabilitation Medicine, Amsterdam UMC, Vrije Universiteit

Amsterdam, Amsterdam Public Health research institute, De Boelelaan 1118,

$1081 \mathrm{HZ}$ Amsterdam, Netherlands

Full list of author information is available at the end of the article
}

(c) The Author(s). 2019 Open Access This article is distributed under the terms of the Creative Commons Attribution 4.0 International License (http://creativecommons.org/licenses/by/4.0/), which permits unrestricted use, distribution, and reproduction in any medium, provided you give appropriate credit to the original author(s) and the source, provide a link to the Creative Commons license, and indicate if changes were made. The Creative Commons Public Domain Dedication waiver (http://creativecommons.org/publicdomain/zero/1.0/) applies to the data made available in this article, unless otherwise stated. 
(Continued from previous page)

Trial registration: The trial is registered at the Dutch Trial Register (NTR5474). Registered 12 October 2015. Retrospectively registered.

Keywords: Trauma, Fractures, Rehabilitation, HR-QOL, Functional outcome, Transmural care, Organization

\section{Background}

Traumatic injury-related mortality accounts for almost $10 \%$ of the global annual mortality. Moreover, major trauma accounts for the highest mortality rate among people under 40 years of age, compared to any other disease $[1,2]$. As a consequence, traumatic injury is responsible for the highest loss of Disability-Adjusted Life Years (DALYs) worldwide. Each year, trauma costs the global population about 300 million years of healthy life, equaling $11 \%$ of DALYs lost [3]. Furthermore, in adults younger than 45 years, major trauma is the most important cause of long-term functional limitations [4].

Many trauma patients have more than one fracture. Fractures, and those of the lower extremities in particular, significantly impact a patient's functional status and health-related quality of life (HR-QOL) [5, 6]. On top of that, the economic burden of trauma to society is extensive, for example, the societal cost of an operatively treated vertebral fracture was estimated at $€ 66.000$ per patient [7]. Furthermore, Fakhry et al. showed that trauma patients represent a significant and increasing institutional cost, of which ICU costs per trauma patient were the largest single category [8]. During the last decades, a significant decrease in mortality has been achieved among severe trauma patients through the optimization of pre-hospital and in-hospital trauma care [9-12]. As further reductions in mortality rates are therefore expected to be trivial, the focus of trauma care has shifted from aiming to reduce mortality rates to aiming to improve trauma patients' HR-QOL and outcome [9-12]. As a consequence, HR-QOL has become one of the most important outcome measures in studies among severely injured trauma patients [13, 14], whereas relatively few studies have focused on measuring HR-QOL amongst mildly to moderately injured patients $[15,16]$.

To further improve outcome and HR-QOL among mild, moderate, and severe trauma patients, increased attention is required for optimizing the rehabilitation process after in-hospital trauma care [17-19]. Research among other patient groups indicates that an improved organization of the post-clinical rehabilitation process can lead to better outcomes [20-22]. For example, a study in patients with Parkinson's disease indicates that a post-clinical care model in which rehabilitation is organized in a network of experienced and specialized healthcare providers results in better clinical outcomes and lower costs compared to regular care models [20].
Furthermore, a feasibility study among patients with hip or knee osteoarthritis found a care model, in which primary care providers were guided by a clinical case manager, to significantly improve patients' outcome and HR-QOL [21].

Given the above, we developed a new Transmural Trauma Care Model (TTCM) for trauma patients. The core of the TTCM is a continuous feedback loop, in which a multidisciplinary hospital-based team supervises a network of primary care physical therapists.

The aim of the current study is to assess the following research questions:

1) What is the effectiveness of the TTCM on HR-QOL (generic- and disease-specific), pain, functional status, patient satisfaction and perceived recovery, compared to regular care, in trauma patients with at least one fracture?

2) What is the recovery pattern of trauma patients receiving the TTCM, during the 9 month follow-up period, regarding HR-QOL (generic- and diseasespecific), pain and functional status?

\section{Methods}

The study protocol of the current study, with detailed descriptions of its design and methods, has been published elsewhere [23]. Alongside the present study (assessing the effectiveness of the TTCM), the cost-effectiveness of the TTCM was evaluated in an economic evaluation, of which the results were recently published [24]. Consequently, some parts of the method section below are overlapping with the aforementioned publications (i.e. patients, inclusion procedure, intervention- and control conditions and outcome measures). An abridged version of the earlier published study protocol, is presented below.

\section{Design}

A modified controlled-before-and-after study was conducted at the outpatient clinic for trauma patients of the Amsterdam UMC, Vrije Universiteit Amsterdam (VUmc), the Netherlands [23, 24]. In a true controlled-before-andafter study both study groups are prospectively followed [25]. However, in the present study, only the intervention group was prospectively followed, while control group data were collected cross-sectionally.

From January to March 2014, control group data were collected among patients who received regular care. The 
control group consisted of 4 clusters of patients. The baseline, 3-month, 6-month, and 9-month clusters contained patients who had their first consultation at the outpatient clinic within 1 week ago, or 3 months ago, 6 months ago, and 9 months ago, respectively. All control group patients were only measured once at the time point that corresponds to the cluster they belong to.

From April to May 2014, the TTCM was implemented. Subsequently, intervention group participants were recruited from June 2014 to April 2015, after which they received care according to the TTCM. All intervention group patients were prospectively followed for 9 months with measurements at baseline and 3, 6 and 9 months after their first consultation at the outpatient clinic. A graphical representation of the study design can be found in Fig. 1 .

The medical ethics committee of the VUmc assessed the present study, and decided that the Dutch Medical Research Involving Human Subjects Act (WMO) was not applicable (registered under number 2013.454). All participants gave informed consent. The trial is registered at the Dutch Trial Register (NTR5474) and adheres to the CONSORT guideline.

\section{Patients}

Operatively and non-operatively treated trauma patients were included, regardless of whether or not they were admitted to the hospital. Eligible patients had to have at least one traumatic fracture (i.e. upper and lower extremity fractures, spinal fractures, hip fractures), had to be 18 years or older, had to rehabilitate in primary care and had to be able to fill out Dutch online questionnaires. Patients were excluded if they had non-traumatic (pathological) fractures, or traumatic brain injury, or cognitive limitations. Furthermore patients were excluded if their rehabilitation occurred in a clinical tertiary care setting, or if they lived more than $30 \mathrm{~km}$ away from the Amsterdam UMC, VUmc.

The recruitment procedure of potential participants took place as earlier described in the study protocol [23, 24]. Control group participants were selected from the central trauma registry of the trauma region "North West Netherlands". All sequential patients were contacted by telephone by one of the investigators and received information about the study's purpose and procedures. In- and exclusion criteria were verified by the principle investigator, after which patients were allocated to their respective cluster. Eligible patients who were willing and able to participate received an email inclosing a link to an online questionnaire. Clicking the link to the online questionnaire served as informed consent. A reminder email was send after 1 week and again after another week of non-responding. In case of patients not replying to both emails, one of the coordinating investigators contacted the patient by telephone.

Intervention group participants were identified during their first consultation at the outpatient clinic as described in the study protocol $[23,24]$. Potentially eligible patients were informed about the study's purpose and

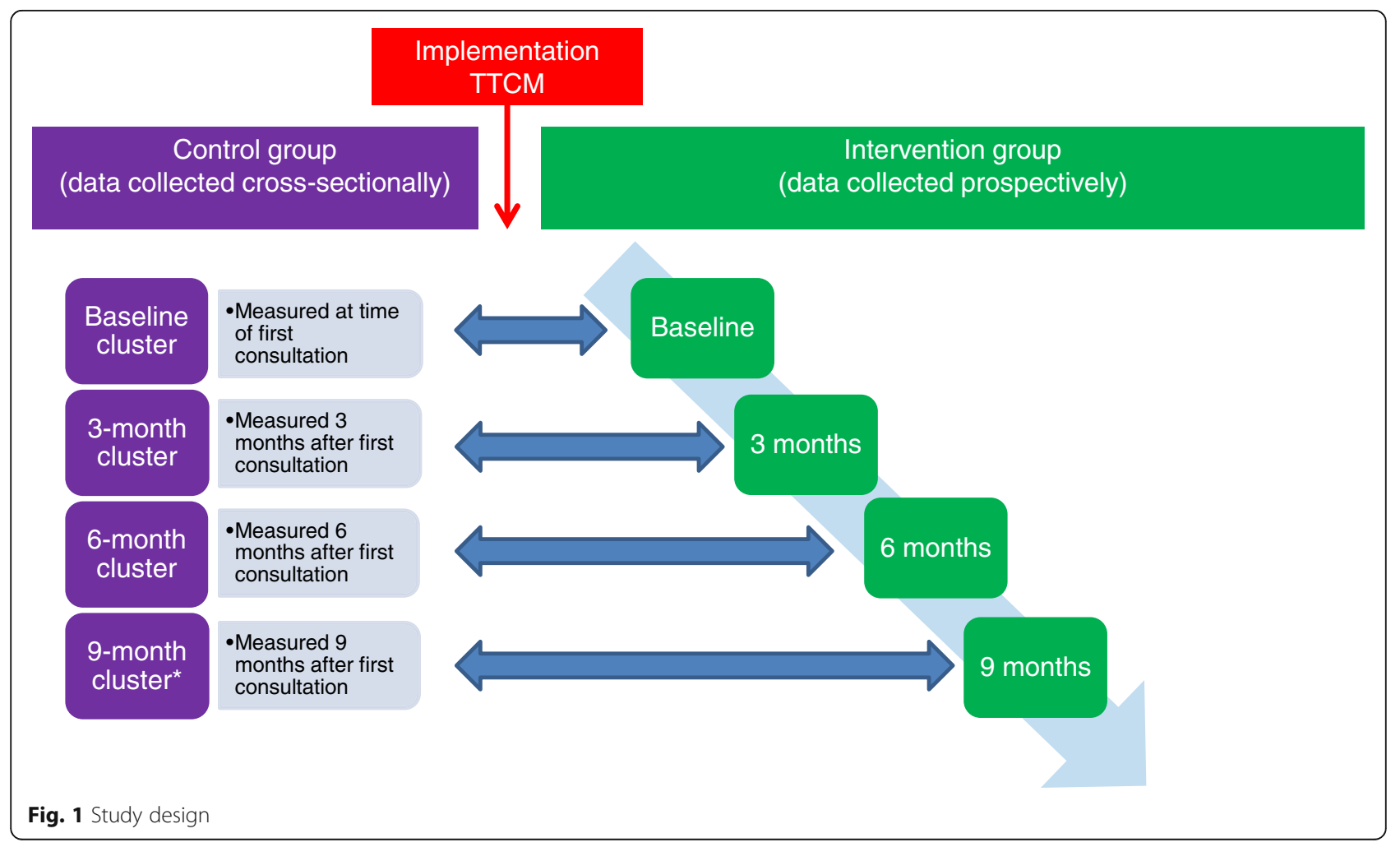


procedures by one of the investigators and in- and exclusion criteria were verified. Eligible patients who were willing and able to participate received an email inclosing a link to the first online questionnaire. Clicking the link to the online questionnaire served as informed consent. A reminder email was send after 1 week and, if necessary, again after another week of non-responding. One of the coordinating investigators contacted the patient by telephone, in case of patient's not replying to both reminder emails. Then, patients were prospectively followed, with measurements at 3, 6, and 9 follow-up.

\section{Intervention conditions}

Pre- and in- hospital trauma care was similar for both study groups, the intervention phase started at the outpatient clinic for trauma patients.

\section{The transmural trauma care model (TTCM)}

Patients in the intervention group received care according to the TTCM. A detailed description of the TTCM can be found elsewhere [23, 24]. In brief, the TTCM consists of four main components:

1) A multidisciplinary team at the outpatient clinic for trauma patients. The team consists of a trauma surgeon and a trauma-specialized hospital-based physical therapist. The trauma surgeon evaluated the bone- and wound healing process. The physical therapist assessed physical function.

2) Coordination and individual goal setting for each patient by the multidisciplinary team. The hospitalbased team coordinated the patients' rehabilitation process in primary care by repeatedly defining individual goals in close cooperation with the patient. To supplement this process, 10 rehabilitation protocols were developed for the most common fractures (e.g. hip fractures, tibial plateau fractures). These protocols were customized for each individual patient by the hospital-based physical therapist, who acted as case manager throughout the rehabilitation process.

3) An educated and trained network of 40 specialized primary care physical therapists. This newly developed "VUmc trauma rehabilitation network" consisted of 40 specially trained, physical therapists, all of whom worked in a primary care private practice in the region of Amsterdam [26]. Patients in the intervention group were referred to one of these specialized trauma physical therapists.

4) Secure email traffic between the hospital-based physical therapist and the primary care physical therapist during the entire rehabilitation process. A secured email system, developed for healthcare professionals, was connected to both the electronic patient records of the hospital-based physical therapist and the primary care physical therapist.

\section{Regular care}

Patients in the control group received regular care, during which the trauma surgeon acted as the chief consultant. The trauma surgeon performed consultations at the outpatient clinic for trauma patients, and acted independent of other health care professionals. Based on the clinical judgment of the trauma surgeon, a patient could be referred to a primary care physical therapist, but there was no standardized policy for referral of control group patients. Throughout the patients' rehabilitation in primary care, there was hardly any contact between trauma surgeon and primary care physical therapists.

\section{Outcome assessment}

An overview of all outcome measurements is provided in Table 1. Extensive details of the outcome measures can be found elsewhere [23, 24].

\section{Baseline characteristics}

At baseline, various relevant demographic and traumarelated characteristics were measured (e.g. gender, age, medical history, ISS, the number of days between trauma and first outpatient consultation [TTO]). Baseline characteristics were collected using online questionnaires, supplemented by data derived from electronic patient records.

\section{Primary outcome measure}

The primary outcome measure was generic HR-QOL, assessed using the EQ-5D-3L [27]. The EQ-5D-3L consists of 5 questions covering 5 health dimensions (i.e. mobility, self-care, usual activities, pain/discomfort and anxiety/depression), all of which contain 3 severity levels. Using the Dutch tariff, the participants' EQ-5D3L health states were converted into a utility score, anchored at 0 (dead) and 1 (optimal health).

\section{Secondary outcome measures}

Secondary outcome measures were disease-specific HRQOL, pain, functional status, patient satisfaction and perceived recovery.

Depending on the patients' specific injury type, disease-specific HR-QOL was measured using one of the following disease-specific function scales:

- The Quick Dash for patients with upper extremity fractures, consisting of 11 items, measuring physical function and symptoms on a five-point scale. The overall score ranges from 0 to $100[28,29]$.

- The Lower Extremity Functional Scale (LEFS) for patients with hip fractures or other lower extremity 
Table 1 Overview of all outcome measurements

\begin{tabular}{|c|c|c|c|c|c|}
\hline Outcomes & $\begin{array}{l}\text { Measurement } \\
\text { Instrument }\end{array}$ & Abbreviation & Items & Itemscore & Interpretation \\
\hline \multicolumn{6}{|l|}{ Primary outcome } \\
\hline Generic HR-QOL & EQ-5D-3L & EQ-5D-3L & 5 & $1-3$ & $\begin{array}{l}\text { higher utility score: better } \\
\text { health }\end{array}$ \\
\hline \multicolumn{6}{|l|}{ Secondary outcomes } \\
\hline Disease-specific HR-QOL (upper extremity) & Quick Dash score & Q-DASH & 11 & $1-5$ & $\begin{array}{l}\text { sumscore } 0-100 \text {, higher score: } \\
\text { less function }\end{array}$ \\
\hline Disease-specific HR-QOL (lower extremity) & $\begin{array}{l}\text { Lower Extremity Functional } \\
\text { Scale }\end{array}$ & LEFS & 20 & $0-4$ & $\begin{array}{l}\text { sumscore } 0-80 \text {, higher score: } \\
\text { better function }\end{array}$ \\
\hline Disease-specific HR-QOL (vertebral fractures) & Roland Morris Disability Score & RMDS & 24 & yes/no & $\begin{array}{l}\text { sumscore } 0-24 \text {, higher score: } \\
\text { more disability }\end{array}$ \\
\hline $\begin{array}{l}\text { Disease-specific HR-QOL (multi trauma } \\
\text { patients) }\end{array}$ & $\begin{array}{l}\text { Groningen Activity Restriction } \\
\text { Scale }\end{array}$ & GARS & 18 & $1-4$ & $\begin{array}{l}\text { sumscore } 18-72 \text {, higher score: } \\
\text { more restrictions }\end{array}$ \\
\hline Disease-specific HR-QOL (over-all) & Q-DASH, LEFS, RMDS, GARS & DSQOL-OA & & & $\begin{array}{l}\text { sumscore } 0-100 \text {, higher score: } \\
\text { less function }\end{array}$ \\
\hline Pain & Numeric Pain Rating Scale & NPRS & 1 & $0-10$ & higher score: more pain \\
\hline Functional status & Patient Specific Function Scale & PSFS & 3 & $\begin{array}{l}100 \mathrm{~mm} \\
\text { VAS }\end{array}$ & higher score: less function \\
\hline Patient satisfaction & Numeric Rating Scale & NRS & 3 & $0-10$ & higher score: more satisfaction \\
\hline Perceived recovery & Global Perceived Effect & GPE & 1 & $1-7$ & higher score: less recovery \\
\hline
\end{tabular}

fractures. The LEFS is a 20-item questionnaire with 5 answering options. The overall score ranges from 0 to $80[30,31]$.

- The Roland Morris Disability Score (RMDS) for patients with vertebral fractures. The RMDS is a 24item questionnaire with 2 answering categories (yes/ no). The overall score ranges from 0 to 24 [32, 33].

- The Groningen Activity Restriction Scale (GARS) for multi trauma patients. The GARS is an 18-item questionnaire with 4 response categories, measuring daily activities. The overall score ranges from 18 to $72[34,35]$.

An overall disease-specific HR-QOL score (DSQOLOA) was calculated by converting the total scores of the aforementioned questionnaires to a scale from 0 to 100 . Higher scores indicated that patients experienced more functional problems [24].

The Numeric Pain Rating Scale (NPRS) was used to measure pain. The NPRS is an 11-point scale ranging from 0 (no pain) to 10 (worst possible pain) [36].

Functional status was measured using the Patient Specific Function Scale (PSFS) [37, 38]. Patients identified 3 important activities that they were having difficulty with. Per activity, they were asked to rate their present level of difficulty associated with each activity on a $0-100 \mathrm{~mm}$ Visual Analogue Scale (VAS) ranging from 0 ("able to perform activity at same level as before injury or problem") to 100 ("unable to perform activity"). The activity that was first mentioned by the participants, was used for statistical analysis.
Patient satisfaction was examined on an 11-point $\mathrm{Nu}$ meric Rating Scale (NRS) ranging from 0 (very dissatisfied) to 10 (excellent). Three patient satisfaction components related to the TTCM were evaluated: 1) the over-all treatment, 2) treatment located at the outpatient clinic, 3) collaboration between the multidisciplinary team at the outpatient clinic and the primary care physical therapist.

Perceived recovery was examined using the Global Perceived Effect (GPE) scale. The GPE quantifies a patient's subjective improvement on a 7-item scale, ranging from "worse than ever" (1) to "completely recovered" (7) [39]. Success of treatment was achieved when a patient reported 6 or 7 points meaning respectively "much improved" or "completely recovered".

\section{Data analysis}

The current data analysis section is highly comparable to the version previously described in the study protocol [23].

\section{Descriptive statistics}

Descriptive statistics were used to compare baseline characteristics between study groups.

\section{Handling missing data}

Missing data were imputed using Multiple Imputation by Chained Equations [40]. An imputation model was built, including variables predicting the outcomes, variables that are related to the "missingness" of data, and furthermore, all available midpoint and follow-up effect measure values [40]. Ten complete data sets were created in order for the loss-of-efficiency to be below 5\% 
[41]. All of the imputed datasets were analysed separately as specified below. Rubin's rules were used to subsequently calculate Pooled estimates [41].

\section{Clinical effectiveness}

The clinical effectiveness analyses contained two parts. First, linear regression analyses were used to investigate the effectiveness of the TTCM in terms of HR-QOL (genericand disease-specific), pain, functional status, patient satisfaction and perceived recovery compared with regular care at 3-, 6- and 9-months follow-up. For this purpose, three clusters of control patients (i.e. 3-month cluster, 6-month cluster, and 9-month cluster) were compared with the patients in the intervention group at the corresponding time points. Second, the recovery patterns of the intervention group patients for generic- and disease-specific HR-QOL, pain and functional status during the 9-month follow-up period was studied using GLM for repeated measures [42]. All analyses were adjusted for confounders if necessary (e.g. gender, fracture region, length of stay). Confounding was examined by adding the potential confounding variable to the crude models. If the regression coefficient changed by

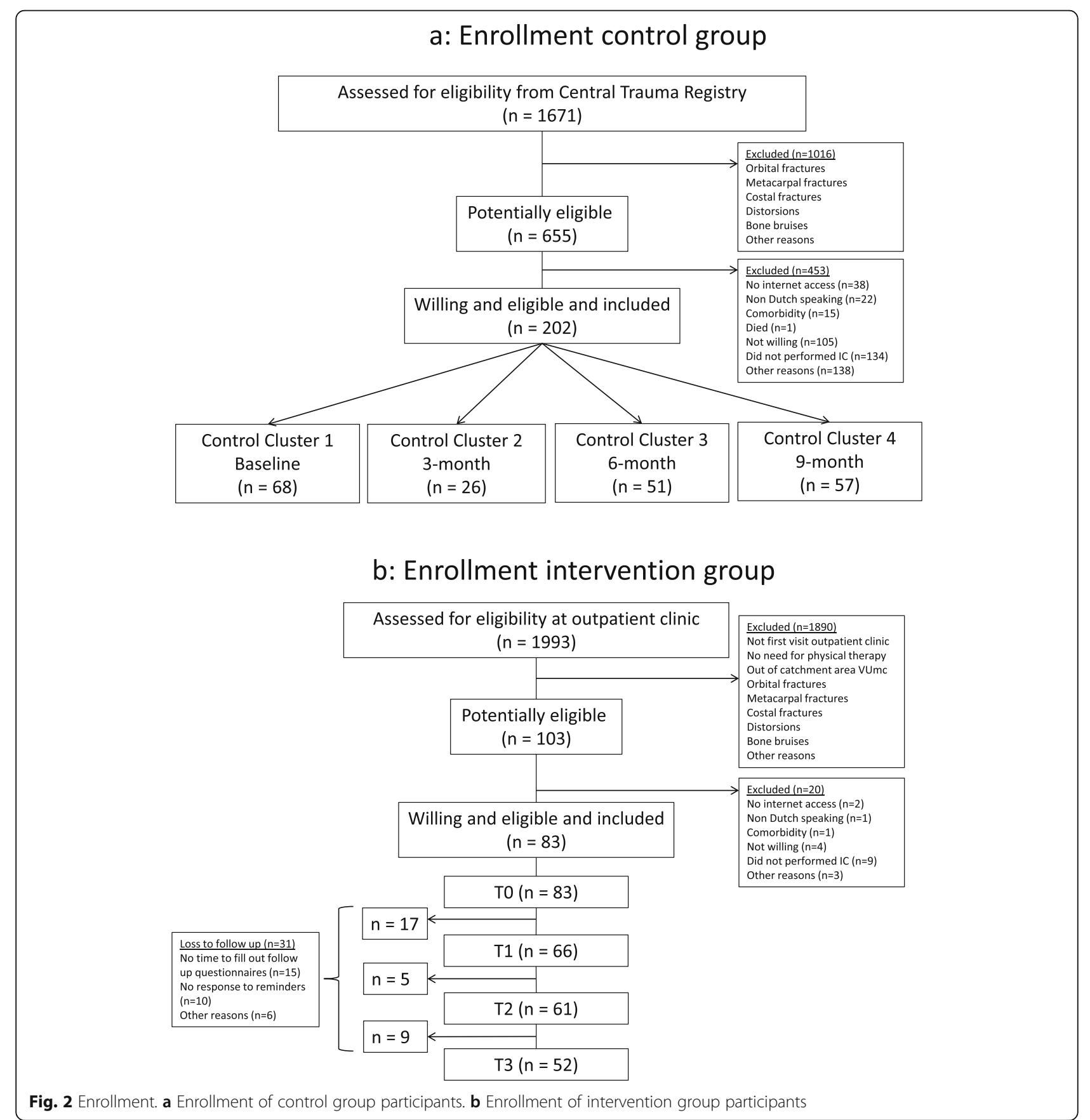


$10 \%$ or more, confounding was considered to be present. Analyses were performed in SPSS Version 22, using a level of significance of $p<0.05$.

\section{Results}

\section{Study participants}

A total of 655 trauma patients were identified as being potentially eligible for participation in the control group. Of them, 453 patients were excluded for various reasons (e.g. did not provide informed consent $(n=134)$, not willing to participate $(n=105))$. The remaining 202 patients were included in the control group, of which 68 in the baseline cluster, 26 in the 3-month cluster, 51 in the 6-month cluster, and 57 in the 9-month cluster (Fig. 2a).
For the intervention group, a total of 103 potentially eligible patients were identified, of whom 20 were eventually excluded for several reasons (e.g. did not provide informed consent $(n=9)$, did not have internet access $(n=2))$. The remaining 83 patients were included as participants in the intervention group (Fig. 2b).

Baseline characteristics of participants in the four control group clusters and the intervention group are described in Table 2. The majority of these characteristics were similar among participants. However, participants in the intervention group were younger, were more frequently admitted to the hospital, and had lower extremity fractures more often than their control group counterparts.

Table 2 Baseline characteristics (patient- and trauma related)

\begin{tabular}{|c|c|c|c|c|c|}
\hline \multirow[t]{2}{*}{ Characteristics } & Intervention group & $\begin{array}{l}\text { Control group } \\
\text { Cluster } 1 \text { (baseline) }\end{array}$ & $\begin{array}{l}\text { Control group } \\
\text { Cluster } 2 \text { (3-month) }\end{array}$ & $\begin{array}{l}\text { Control group } \\
\text { Cluster } 3 \text { (6-month) }\end{array}$ & $\begin{array}{l}\text { Control group } \\
\text { Cluster } 4 \text { (9-month) }\end{array}$ \\
\hline & $\begin{array}{l}\text { Mean (SD) or frequency } \\
(\%)\end{array}$ & $\begin{array}{l}\text { Mean (SD) or frequency } \\
(\%)\end{array}$ & $\begin{array}{l}\text { Mean (SD) or frequency } \\
(\%)\end{array}$ & $\begin{array}{l}\text { Mean (SD) or frequency } \\
(\%)\end{array}$ & $\begin{array}{l}\text { Mean (SD) or frequency } \\
(\%)\end{array}$ \\
\hline $\mathrm{N}$ & 83 & 68 & 26 & 51 & 57 \\
\hline Age & $43.4(15.6)$ & $46.8(14.3)$ & $57.2(16.0)$ & $50.0(17.4)$ & $50.5(17.9)$ \\
\hline Gender (M/F) & $39 / 44$ (47/53\%) & $31 / 37$ (46/54\%) & $13 / 13(50 / 50 \%)$ & $22 / 29(43 / 57 \%)$ & 26/31 (46/54\%) \\
\hline \multicolumn{6}{|l|}{ Education level } \\
\hline Low & $7(8.4 \%)$ & $12(18.5 \%)$ & $2(8.3 \%)$ & $5(10.6 \%)$ & $6(11.1 \%)$ \\
\hline Middle & 19 (22.9\%) & $16(24.6 \%)$ & $4(16.7 \%)$ & $14(29.8 \%)$ & $16(29.6 \%)$ \\
\hline High & 57 (68.7\%) & 37 (56.9\%) & $18(75.0 \%)$ & $28(59.6 \%)$ & $32(59.3 \%)$ \\
\hline \multicolumn{6}{|l|}{ Medical history } \\
\hline None & $53(63.9 \%)$ & $33(48.5 \%)$ & $14(53.8 \%)$ & $30(60.0 \%)$ & $30(52.6 \%)$ \\
\hline Chronic & $14(16.9 \%)$ & $21(30.9 \%)$ & $7(26.9 \%)$ & $9(18 \%)$ & $13(22.8 \%)$ \\
\hline Musculoskeletal & $16(19.3 \%)$ & $14(20.6 \%)$ & $5(19.2 \%)$ & $11(22 \%)$ & $14(24.6 \%)$ \\
\hline \multicolumn{6}{|l|}{ Trauma type } \\
\hline Traffic & $44(53.0 \%)$ & $26(38.8 \%)$ & $9(34.6 \%)$ & $15(29.4 \%)$ & $25(43.9 \%)$ \\
\hline Work related & 0 & $3(4.5 \%)$ & $3(11.5 \%)$ & $2(3.9 \%)$ & $2(3.5 \%)$ \\
\hline Fall & 27 (32.5\%) & $13(19.4 \%)$ & $9(34.6 \%)$ & $20(39.2 \%)$ & 17 (29.8\%) \\
\hline Sports & $11(13.3 \%)$ & $19(28.4)$ & $5(19.2 \%)$ & $9(17.6 \%)$ & $9(15.8 \%)$ \\
\hline Other & $1(1.2 \%)$ & $6(9 \%)$ & 0 & $5(9.8 \%)$ & $4(7.0 \%)$ \\
\hline \multicolumn{6}{|l|}{ Fracture region } \\
\hline Upper extremity & $31(37.3 \%)$ & $33(48.5 \%)$ & $14(53.8 \%)$ & $25(49.0 \%)$ & $25(43.9 \%)$ \\
\hline Lower extremity & $41(49.4 \%)$ & $25(36.8 \%)$ & $9(34.6 \%)$ & $16(31.4 \%)$ & 19 (33.0\%) \\
\hline Vertebral & $7(8.4 \%)$ & 0 & $1(3.8 \%)$ & $2(3.9 \%)$ & $1(1.8 \%)$ \\
\hline Multitrauma & $4(4.8 \%)$ & $10(14.7 \%)$ & $2(7.7 \%)$ & $8(15.7 \%)$ & $12(21.1 \%)$ \\
\hline ISS (mean, SD) & $7.9(4.4)$ & $9.1(6.6)$ & $7.7(5.6)$ & $8.9(7.8)$ & $8.6(6.3)$ \\
\hline ISS (min-max) & $4-26$ & $4-34$ & $4-24$ & $4-43$ & $4-29$ \\
\hline Admission hospital & $62(75 \%)$ & $44(65 \%)$ & $8(31 \%)$ & $24(47 \%)$ & $29(51 \%)$ \\
\hline $\begin{array}{l}\text { Length of stay } \\
\text { (days) }\end{array}$ & $7.1(6.1)$ & $8.2(7.3)$ & $8.4(11.1)$ & $10.8(8.0)$ & 10.0 (11.4) \\
\hline Surgery & $53(64 \%)$ & $36(53 \%)$ & $5(19 \%)$ & $22(43 \%)$ & $21(37 \%)$ \\
\hline TTO (days) ${ }^{\mathrm{a}}$ & $24.3(14.3)$ & $15.9(13.1)$ & 11.5 (15.9) & $16.0(15.8)$ & $14.6(14.7)$ \\
\hline
\end{tabular}


Table 3 Treatment effects for primary and secondary outcomes

\begin{tabular}{|c|c|c|c|c|c|}
\hline Outcomes & $\begin{array}{l}\text { Intervention group } \\
\text { Mean (SD) }\end{array}$ & $\begin{array}{l}\text { Control group } \\
\text { Mean (SD) }\end{array}$ & $\begin{array}{l}\text { Treatment effect } \\
\text { (crude) MD } \\
(95 \% \mathrm{Cl})\end{array}$ & $\begin{array}{l}\text { Treatment effect } \\
\text { (adjusted) MD } \\
(95 \% \mathrm{Cl})\end{array}$ & Adjusted for ${ }^{a}$ \\
\hline \multicolumn{6}{|c|}{ Primary outcome } \\
\hline \multicolumn{6}{|c|}{ HR-QOL (EQ-5D-3L) } \\
\hline Baseline & $0.65(0.21)$ & $0.70(0.19)$ & $-0.05(-0.11-0.021)$ & $-0.031(-0.1-0.038)$ & $\begin{array}{l}\text { age, } T O^{b} \text {, admission hospital, } \\
\text { surgery }\end{array}$ \\
\hline 3 months & $0.78(0.16)$ & $0.85(0.13)$ & $-0.07(-0.14-0.006)$ & $-0.083(-0.17-0.001)$ & age, $T \mathrm{TO}$, fracture region \\
\hline 6 months & $0.82(0.13)$ & $0.81(0.19)$ & $0.013(-0.05-0.08)$ & $0.051(-0.02-0.12)$ & $\begin{array}{l}\text { gender, trauma type, TTO, } \\
\text { admission hospital, surgery }\end{array}$ \\
\hline 9 months & $0.85(0.13)$ & $0.81(0.19)$ & $0.04(-0.02-0.10)$ & $0.055(-0.01-0.12)$ & age, medical history, ТTO \\
\hline \multicolumn{6}{|c|}{ Secondary outcomes } \\
\hline \multicolumn{6}{|c|}{ Disease-specific HR-QOL (DSQOL-OA) } \\
\hline Baseline & $55.60(21.35)$ & $50.61(22.26)$ & $4.99(-1.88-11.86)$ & $3.65(-3.37-10.67)$ & $\begin{array}{l}\text { age, medical history, } \Pi T O \text {, fracture } \\
\text { region, admission hospital, surgery }\end{array}$ \\
\hline 3 months & $29.56(20.06)$ & $26.16(23.35)$ & $3.44(-5.21-12.09)$ & $0.36(-8.85-9.58)$ & $\begin{array}{l}\text { age, medical history, fracture region, } \\
\text { admission hospital, surgery, } \\
\text { length of stay }\end{array}$ \\
\hline 6 months & $22.66(20.01)$ & $20.21(20.09)$ & $2.45(-3.91-8.82)$ & $-3.65(-10.38-3.08)$ & $\begin{array}{l}\text { age, medical history, trauma type, TTO, } \\
\text { fracture region, ISS, admission hospital, } \\
\text { surgery }\end{array}$ \\
\hline 9 months & $17.63(15.74)$ & $20.45(21.00)$ & $-2.82(-8.41-2.77)$ & $-7.96(-14.17--1.75)$ & $\begin{array}{l}\text { age, medical history, TTO, fracture } \\
\text { region, admission hospital, surgery }\end{array}$ \\
\hline \multicolumn{6}{|l|}{ Pain (NPRS) } \\
\hline Baseline & $2.84(1.85)$ & $2.83(1.93)$ & $0.013(-0.60-0.62)$ & $0.34(-0.36-1.04)$ & $\begin{array}{l}\text { gender, age, education, medical history, } \\
\text { trauma type, TTO, fracture region, ISS, } \\
\text { admission hospital, surgery }\end{array}$ \\
\hline 3 months & $1.95(1.26)$ & $2.54(1.64)$ & $-0.59(-1.2-0.02)$ & $-0.38(-1.07-0.32)$ & TTO, admission hospital, surgery \\
\hline 6 months & $1.63(1.33)$ & $2.31(2.09)$ & $-0.68(-1.26--0.11)$ & $-0.87(-1.44--0.29)$ & trauma type \\
\hline 9 months & $1.34(1.11)$ & $2.19(2.01)$ & $-0.84(-1.38--0.31)$ & $-0.84(-1.38--0.31)$ & none \\
\hline \multicolumn{6}{|c|}{ Functional status (PSFS) } \\
\hline Baseline & $65.9(28.89)$ & $58.15(37.1)$ & $6.84(-3.69-17.37)$ & $5.90(-5.45-17.24)$ & education, medical history, fracture region \\
\hline 3 months & $26.71(26.82)$ & $34.00(35.72)$ & $-7.29(-19.32-4.74)$ & $-2.02(-15.82-11.76)$ & $\begin{array}{l}\text { medical history, trauma type, TTO, } \\
\text { fracture region, admission hospital, } \\
\text { length of stay }\end{array}$ \\
\hline 6 months & $18.49(22.06)$ & $29.64(25.43)$ & $-11.15(-18.66--3.64)$ & $-16.49(-24.39--8.60)$ & $\begin{array}{l}\text { trauma type, TTO, admission hospital, } \\
\text { surgery }\end{array}$ \\
\hline 9 months & $18.39(22.36)$ & $34.39(30.74)$ & $-16.00(-24.09--7.90)$ & $-20.68(-29.20--12.16)$ & TTO, surgery \\
\hline \multicolumn{6}{|c|}{ Patient satisfaction (total treatment) } \\
\hline Baseline & $7.84(1.48)$ & $7.32(2.01)$ & $0.53(-0.03-1.08)$ & $0.60(0.036-1.16)$ & education \\
\hline 3 months & $8.10(0.99)$ & $7.17(1.43)$ & $0.93(0.38-1.47)$ & $0.77(0.13-1.42)$ & $\begin{array}{l}\text { TTO, admission hospital, surgery, } \\
\text { length of stay }\end{array}$ \\
\hline 6 months & $8.09(1.35)$ & $7.44(2.04)$ & $0.64(0.07-1.22)$ & $0.53(-0.07-1.13)$ & admission hospital \\
\hline 9 months & $8.09(1.57)$ & $7.77(1.18)$ & $0.32(-0.19-0.82)$ & $0.24(-0.29-0.76)$ & surgery \\
\hline \multicolumn{6}{|c|}{ Patient satisfaction (outpatient clinic) } \\
\hline Baseline & $7.76(1.57)$ & $7.33(2.01)$ & $0.43(-0.15-1.00)$ & $0.56(-0.018-1.15)$ & education, medical history \\
\hline 3 months & $7.73(1.55)$ & $7.41(1.66)$ & $0.31(-0.37-1.00)$ & $0.15(-0.64-0.95)$ & $\begin{array}{l}\text { medical history, TTO, fracture } \\
\text { region, admission hospital, } \\
\text { surgery, length of stay }\end{array}$ \\
\hline 6 months & $7.98(1.46)$ & $7.50(1.92)$ & $0.48(-0.09-1.05)$ & $0.39(-0.25-1.03)$ & $\begin{array}{l}\text { trauma type, fracture region, } \\
\text { admission hospital, surgery }\end{array}$ \\
\hline 9 months & $8.22(1.41)$ & $7.71(1.36)$ & $0.51(0.03-0.98)$ & $0.44(-0.08-0.95)$ & age, $\Pi \mathrm{TO}$, surgery \\
\hline
\end{tabular}


Table 3 Treatment effects for primary and secondary outcomes (Continued)

\begin{tabular}{|c|c|c|c|c|c|}
\hline Outcomes & $\begin{array}{l}\text { Intervention group } \\
\text { Mean (SD) }\end{array}$ & $\begin{array}{l}\text { Control group } \\
\text { Mean (SD) }\end{array}$ & $\begin{array}{l}\text { Treatment effect } \\
\text { (crude) MD } \\
\text { (95\% Cl) }\end{array}$ & $\begin{array}{l}\text { Treatment effect } \\
\text { (adjusted) MD } \\
\text { (95\% Cl) }\end{array}$ & Adjusted for ${ }^{a}$ \\
\hline \multicolumn{6}{|c|}{ Patient satisfaction (collaboration) } \\
\hline Baseline & $7.16(2.00)$ & $5.77(2.57)$ & $1.39(0.39-2.39)$ & $1.25(0.24-2.25)$ & trauma type \\
\hline 3 months & $7.40(1.31)$ & $5.61(2.19)$ & $1.79(0.93-2.66)$ & $1.61(0.72-2.51)$ & ТТО \\
\hline 6 months & $7.65(1.53)$ & $5.86(2.48)$ & $1.78(1.03-2.53)$ & $1.78(1.03-2.53)$ & none \\
\hline 9 months & $7.51(2.03)$ & $6.13(2.35)$ & $1.39(0.65-2.12)$ & $1.20(0.42-1.97)$ & admission hospital, surgery \\
\hline
\end{tabular}

${ }^{a}$ The baseline characteristics mentioned in this column were confounders (changed the regression coefficient with $10 \%$ or more)

${ }^{\mathrm{b}}$ TTO Time between Trauma and first Outpatient consultation (days)

\section{Clinical effects}

There were no relevant between-group differences in the dependent variable generic HR-QOL (primary outcome measure) at all measurement points (Table 3). However, the mean between-group difference in the dependent variable disease-specific HR-QOL was statistically significant in favor of the intervention group at 9 months (MD -7.96; 95\% CI - 14.17 to -1.75 ), but not at 3 and 6 months. Patients in the intervention group had statistically significant less pain at 6 (MD -0.87; 95\% CI - 1.44 to -0.29 ) and 9 months (MD $-0.84 ; 95 \%$ CI -1.38 to 0.31 ) than their control group counterparts, but no difference in pain was found at 3 months. There was also a statistically significant difference in functional status favoring the intervention group at 6 months (MD -16.49; $95 \% \mathrm{CI}-24.39$ to -8.60 ) and 9 months (MD -20.68; $95 \%$ CI -29.20 to -12.16 ), but not at 3 months. Furthermore, participants in the intervention group were statistically significant more satisfied with their total treatment at 3 months (MD 0.77; 95\% CI 0.13 to 1.42), but not at 6 and 9 months. At all of the time points, patients in the intervention group were statistically significant more satisfied with the collaboration between primary and secondary care (3 months $=$ MD 1.61; 95\% CI 0.72 to $2.51,6$ months $=$ MD $1.78 ; 95 \% \mathrm{CI} 1.03$ to 2.53, and 9 months $=$ MD 1.20; 95\% CI 0.42 to 1.97). However, no statistically significant differences were found at any time point for patient satisfaction regarding the treatment at the outpatient clinic (Table 3).

Based on the Global Perceived Effect, 74.6, 78.3, and $84.6 \%$ of the intervention group patients were "completely recovered" or "much improved" at 3, 6, and 9 months, respectively. Of the control group patients, 53.8, 53.3 and $75 \%$ were "completely recovered" or "much improved" at these time points. At 6 months this effect was statistically significant (OR 3.35; 95\% CI 1.32 to 8.49) (Table 4).

\section{Recovery pattern of patients in the intervention group} During the nine-month follow-up period, generic HRQOL $(\mathrm{F}=18.43 ; p=0.000)$, disease-specific HR-QOL $(\mathrm{F}=6.18 ; p=0.001)$, pain $(\mathrm{F}=17.16 ; p=0.000)$, and functional status $(\mathrm{F}=65.05 ; p=0.000)$ statistically significantly improved in a linear fashion among intervention group patients (Fig. 3a, b, c and d).

\section{Discussion}

Traumatic injury poses a substantial economic burden to society. However, little is currently known about how to optimally organize the post-clinical rehabilitation process of trauma patients. Therefore, the current study developed and evaluated the TTCM, the first transmural care model for the rehabilitation of trauma patients in primary care $[9,10,23]$.

\section{Important study findings and comparison with the literature}

Our results indicate that the TTCM statistically significantly improved disease-specific HR-QOL, pain, functional status, patient satisfaction and perceived recovery among mild, moderate and severe trauma patients. It is important to mention, however, that even though no statistically significant effects were found for generic HRQOL, the identified mean difference can be regarded as clinically relevant at 6 months (MD $0.051 ; 95 \%$ CI -0.02

Table 4 Treatment effect for global perceived effect ("completely recovered" or "much improved")

\begin{tabular}{llllll}
\hline GPE Succes & $\begin{array}{l}\text { Succes\% Intervention } \\
\text { group }\end{array}$ & $\begin{array}{l}\text { Succes\% Control } \\
\text { group }\end{array}$ & $\begin{array}{l}\text { Treatment effect (crude) } \\
\text { OR }(95 \% \text { Cl) }\end{array}$ & $\begin{array}{l}\text { Treatment effect (adjusted) } \\
\text { OR (95\% Cl) }\end{array}$ & Adjusted for $^{\text {a }}$ \\
\hline 3 months & 74.6 & 53.8 & $2.37(0.85-6.62)$ & $2.39(0.69-8.20)$ & $\begin{array}{l}\text { age, fracture region, } \\
\text { length of stay }\end{array}$ \\
$\begin{array}{llll}6 \text { months } \\
9 \text { months }\end{array}$ & 78.3 & 53.3 & $2.99(1.24-7.23)$ & $3.35(1.32-8.49)$ & fracture region \\
\hline
\end{tabular}

${ }^{a}$ The baseline characteristics mentioned in this column were confounders (changed the regression coefficient with $10 \%$ or more)

${ }^{\mathrm{b}}$ TTO Time between Trauma and first Outpatient consultation (days) 


\section{Longitudinal follow up intervention group}
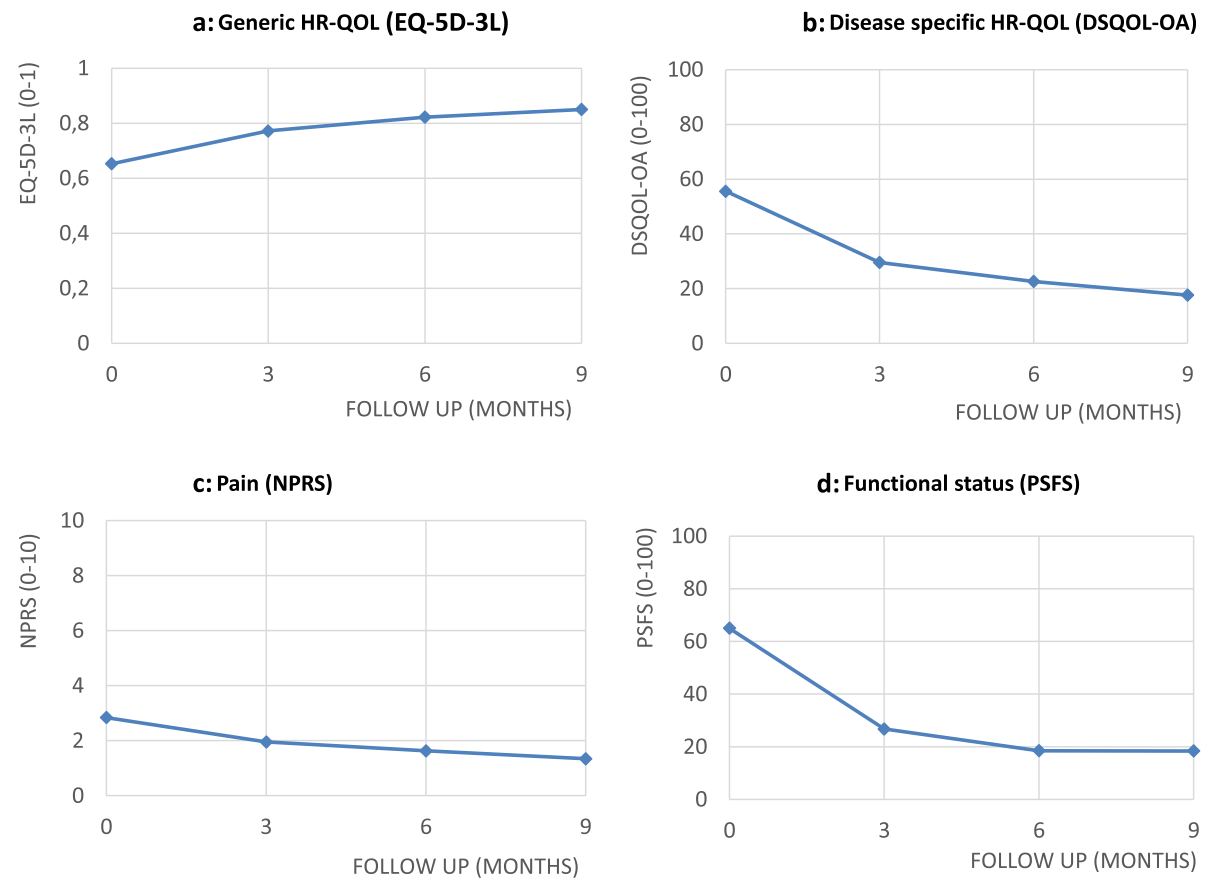

Fig. 3 Longitudinal follow up intervention group. a Generic HR-QOL (EQ-5D-3L). b Disease specific HR-QOL (DSQOL-OA). c Pain (NPRS). d Functional status (PSFS)

to 0.12 ) and 9 months (MD 0.055; $95 \%$ CI -0.01 to 0.12 ). To illustrate, estimates of the minimal clinical important difference (MCID) for the EQ-5D range from 0.03 among patients with low back pain [43] to 0.52 in patients with recurrent lumbar stenosis [44]. In light of this finding it is also important to bear in mind that the current study was not powered to detect a clinically meaningful difference in generic HR-QOL due to its explorative nature.

\section{Strengths and limitations}

The present study population covers a broad range in trauma patients, with an ISS ranging from 4 to 43 . This is an important strength, as the majority of studies only included major trauma patients with an ISS > 16 [4, 14, 45]. Since our study population includes mild, moderate and severely injured patients, the TTCM is likely to be effective in the entire group of trauma patients. However, future research is necessary to examine whether certain subgroups of trauma patients respond in different ways to the TTCM than others.

A second important strength of this study is its clinical relevance as well as the fact that it was the first to develop and evaluate a transmural care model for the postclinical rehabilitation of trauma patients. Other strengths include its use of a broad spectrum of measurement instruments, covering all domains of the International
Classification of Functioning, Disability and Health (ICF) [46], its use of validated questionnaires, as well as its pragmatic design (i.e. daily practice was resembled as much as possible).

The study also had some limitations. Even though the applied modified controlled-before-and-after design was regarded as the most optimal research design within the available resources, it is susceptible to many kinds of bias. Examples of such kinds of bias are selection bias, recall bias, regression to the mean, the Hawthorne effect, and repeat testing bias. Of them, selection bias is probably most likely, meaning that the study groups have a different composition regarding various etiological factors. A multicenter randomized controlled trial would therefore be the next step in order to study the TTCM's effectiveness more robustly. In spite of the fact the all participating trauma patients met the same inclusion criteria, we observed some baseline differences in age (intervention group patients were younger) and admission to hospital (75\% of intervention group patients were admitted to the hospital, compared to $51 \%$ in control group). Based on the recommendations of de Boer et al. we decided not to statistically test baseline differences across study groups [47]. They postulate that statistically testing of baseline differences ignores the fact that the prognostic strength of a variable is also important when the interest is in e.g. adjustment for 
confounding. On top of that, our study was not powered to detect relevant differences at baseline, so possibly relevant differences may also turn out not to be statistically significant. Nonetheless, if we found the addition of a certain baseline variable to change the regression coefficient by more than $10 \%$, they were added to the final models. Another potential limitation was the absence of a sample size calculation. We based the sample size on our estimate of the number of patients that could potentially be included within the time frame and financial constraints of this study. A sample size calculation is preferable in future research, to make sure that the study is not underpowered to detect clinically meaningful effect differences. Another limitation is the fact that we were not able to identify what components of the TTCM were responsible for the positive effects. To illustrate, the better functional outcomes could be the result of an improved communication strategy between the multidisciplinary hospital team and the primary care physical therapist. On the other hand, the better outcomes may have been the result of a better educated and more experienced network of primary care physical therapists. It would be interesting to identify the critical ingredient of this relatively complex intervention. One might also argue, however, that the sum is greater than the individual parts and therefore there is probably no such thing as a critical ingredient. Future research can possibly provide more insight into whether separate TTCM components are accountable for specific effects.

\section{Conclusions}

This study provides preliminary evidence that the TTCM is effective in improving patient-related outcome measures, such as disease-specific HR-QOL and functional status. A multicenter, and ideally randomized controlled trial, is required to confirm these results.

\section{Abbreviations \\ DALY: Disability-Adjusted Life Years; DSQOL-OA: Overall disease-specific HR- QOL; GARS: Groningen Activity Restriction Scale; GPE: Global Perceived Effect; HR-QOL: Health-Related Quality Of Life; ICF: International Classification of Functioning, Disability and Health; ISS: Injury Severity Score; LEFS: Lower Extremity Functional Scale; MCID: Minimal Clinical Important Difference; NRS: Numeric Rating Scale; NTR: The Netherlands National Trial Register; PSFS: Patient Specific Function Scale; RMDS: Roland Morris Disability Score; TTCM: Transmural Trauma Care Model; TTO: Time between Trauma and first Outpatient consultation; VUmc: Amsterdam UMC, Vrije Universiteit Amsterdam}

\section{Acknowledgements}

We thank all trauma patients who participated in the study. Furthermore we thank the participating trauma surgeons and physical therapists for their willingness to change their approach.

\section{Authors' contributions}

SHW has been involved in the development of the TTCM, the design of the study, the implementation of the TTCM, the data collection, the data analysis and writing the manuscript. JMD was closely involved in the design of the study, the data analysis and writing the manuscript. EG was substantially involved in development of the TTCM and was active in finding sources. HB was involved in the data collection of the control group and supervised participating students in the study. FWB, RWO and VG were involved in the overall design of the study and were critically reading the manuscript for important intellectual content. All authors read and approved the final manuscript.

\section{Funding}

This project is partly funded by Achmea, meanwhile known as "Zilveren Kruis Health Insurer" (grant number Z516). The funder had no role in the design of the study, nor in collection, analysis, and interpretation of the data, nor in writing the manuscript.

\section{Availability of data and materials \\ The datasets generated and analysed during the current study are not publicly available due to the volume, but are available from the corresponding author on reasonable request.}

\section{Ethics approval and consent to participate}

The medical ethics committee of the Amsterdam UMC, Vrije Universiteit Amsterdam, assessed the present study, and decided the Dutch Medical Research Involving Human Subjects Act (WMO) was not applicable (registered under number 2013.454). All participants gave electronic informed consent by clicking the link to the online questionnaire in an email they received. This informed consent procedure was approved by the medical ethics committee of the Amsterdam UMC, Vrije Universiteit Amsterdam, since the Dutch Medical Research Involving Human Subjects Act (WMO) was not applicable. The trial is registered at the Dutch Trial Register (NTR5474) and adheres to CONSORT guidelines.

\section{Consent for publication}

Not applicable.

\section{Competing interests}

The authors declare that they have no competing interests.

\section{Author details}

'Department of Rehabilitation Medicine, Amsterdam UMC, Vrije Universiteit Amsterdam, Amsterdam Public Health research institute, De Boelelaan 1118 , 1081 HZ Amsterdam, Netherlands. ${ }^{2}$ Department of Health Sciences, Vrije Universiteit Amsterdam, Amsterdam Public Health research institute, Amsterdam, Netherlands. ${ }^{3}$ Department of Rehabilitation Medicine, Amsterdam UMC, Vrije Universiteit Amsterdam, Amsterdam, Netherlands. ${ }^{4}$ Department of Trauma Surgery, Amsterdam UMC, Vrije Universiteit Amsterdam, Amsterdam, Netherlands. ${ }^{5}$ Department of Epidemiology and Biostatistics, Department of Health Science, Amsterdam UMC, Vrije Universiteit Amsterdam, Amsterdam Public Health research institute, Amsterdam, Netherlands.

Received: 14 March 2019 Accepted: 20 September 2019

Published online: 08 November 2019

\section{References}

1. World Health Organisation. The world health report 2002 - reducing risks, promoting healthy life. Geneva: WHO; 2002.

2. Lozano R, Naghavi M, Foreman K, Lim S, Shibuya K, Aboyans V, Abraham J, Adair T, Aggarwal R, Ahn SY, et al. Global and regional mortality from 235 causes of death for 20 age groups in 1990 and 2010: a systematic analysis for the global burden of disease study 2010. Lancet. 2012;380:2095-128.

3. Murray CJ, Vos T, Lozano R, Naghavi M, Flaxman AD, Michaud C, Ezzati M, Shibuya K, Salomon JA, Abdalla S, et al. Disability-adjusted life years (DALYS) for 291 diseases and injuries in 21 regions, 1990-2010: a systematic analysis for the global burden of disease study 2010. Lancet. 2012;380:2197-223.

4. Holbrook TL, Anderson JP, Sieber WJ, Browner D, Hoyt DB. Outcome after major trauma: 12-month and 18-month follow-up results from the trauma recovery project. J Trauma. 1999:46:765-71 discussion 771-763.

5. Stalp M, Koch C, Ruchholtz S, Regel G, Panzica M, Krettek C, Pape HC. Standardized outcome evaluation after blunt multiple injuries by scoring systems: a clinical follow-up investigation 2 years after injury. J Trauma. 2002;52:1160-8

6. van der Sluis CK, Eisma WH, Groothoff JW, Ten Duis HJ. Long-term physical, psychological and social consequences of a fracture of the ankle. Injury. 1998:29:277-80 
7. Siebenga J, Segers MJ, Leferink VJ, Elzinga MJ, Bakker FC, Duis HJ, Rommens PM, Patka P. Cost-effectiveness of the treatment of traumatic thoracolumbar spine fractures: nonsurgical or surgical therapy? Indian J Orthop. 2007;41:332-6.

8. Fakhry SM, Martin B, Al Harakeh H, Norcross ED, Ferguson PL. Proportional costs in trauma and acute care surgery patients: dominant role of intensive care unit costs. J Am Coll Surg. 2013;216:607-14 discussion 614-606.

9. Lansink KW, Leenen LP. Do designated trauma systems improve outcome? Curr Opin Crit Care. 2007;13:686-90.

10. MacKenzie EJ, Rivara FP, Jurkovich GJ, Nathens AB, Frey KP, Egleston BL, Salkever DS, Scharfstein DO. A national evaluation of the effect of traumacenter care on mortality. N Engl J Med. 2006;354:366-78.

11. Haas B, Jurkovich GJ, Wang J, Rivara FP, Mackenzie EJ, Nathens AB. Survival advantage in trauma centers: expeditious intervention or experience? J Am Coll Surg. 2009;208:28-36.

12. Celso B, Tepas J, Langland-Orban B, Pracht E, Papa L, Lottenberg L, Flint L. A systematic review and meta-analysis comparing outcome of severely injured patients treated in trauma centers following the establishment of trauma systems. J Trauma. 2006;60:371-8 discussion 378.

13. Ardolino A, Sleat G, Willett K. Outcome measurements in major traumaresults of a consensus meeting. Injury. 2012;43:1662-6.

14. Gabbe BJ, Sutherland AM, Hart MJ, Cameron PA. Population-based capture of long-term functional and quality of life outcomes after major trauma: the experiences of the Victorian state trauma registry. J Trauma. 2010;69:532-6 discussion 536.

15. Innocenti F, Del Taglia B, Coppa A, Trausi F, Conti A, Zanobetti M, Pini R. Quality of life after mild to moderate trauma. Injury. 2015;46:902-8.

16. Toien K, Bredal IS, Skogstad L, Myhren H, Ekeberg O. Health related quality of life in trauma patients. Data from a one-year follow up study compared with the general population. Scand J Trauma Resusc Emerg Med. 2011;19:22.

17. Mock C, MacKenzie E, Jurkovich G, Burgess A, Cushing B, deLateur B, McAndrew M, Morris J, Swiontkowski M. Determinants of disability after lower extremity fracture. J Trauma. 2000;49:1002-11.

18. Kempen Gl, Scaf-Klomp W, Ranchor AV, Sanderman R, Ormel J. Social predictors of recovery in late middle-aged and older persons after injury to the extremities: a prospective study. J Gerontol B Psychol Sci Soc Sci. 2001;56:S229-36.

19. Franche RL, Krause N. Readiness for return to work following injury or illness: conceptualizing the interpersonal impact of health care, workplace, and insurance factors. J Occup Rehabil. 2002;12:233-56.

20. Munneke M, Nijkrake MJ, Keus SH, Kwakkel G, Berendse HW, Roos RA, Borm GF, Adang EM, Overeem S, Bloem BR, ParkinsonNet Trial Study G. Efficacy of community-based physiotherapy networks for patients with Parkinson's disease: a cluster-randomised trial. Lancet Neurol. 2010;9:46-54.

21. Voorn VM, Vermeulen HM, Nelissen RG, Kloppenburg M, Huizinga TW, Leijerzapf NA, Kroon HM, Vliet Vlieland TP, van der Linden HM. An innovative care model coordinated by a physical therapist and nurse practitioner for osteoarthritis of the hip and knee in specialist care: a prospective study. Rheumatol Int. 2013;33:1821-8.

22. de Rooij M, van der Leeden $M$, Cheung J, van der Esch M, Hakkinen A Haverkamp D, Roorda LD, Twisk J, Vollebregt J, Lems WF, Dekker J. Efficacy of tailored exercise therapy on physical functioning in patients with knee osteoarthritis and comorbidity: a randomized controlled trial. Arthritis Care Res (Hoboken). 2016;69:807-16.

23. Wiertsema SH, van Dongen JM, Geleijn E, Schothorst M, Bloemers FW, de Groot V, Ostelo RW. Evaluation of a new Transmural trauma care model (TTCM) for the rehabilitation of trauma patients: a study protocol. BMC Health Serv Res. 2017;17:99.

24. Wiertsema SH, van Dongen JM, Geleijn E, Huijsmans RJ, Bloemers FW, de Groot V, Ostelo RW. Cost-effectiveness of the Transmural trauma care model (TTCM) for the rehabilitation of trauma patients. Int J Technol Assess Health Care. 2019:35:307-16.

25. Higgins JTP, Green S. Cochrane handbook for systematic reviews of interventions. Version 5.1.0 [Updated March 2011]. The Cochrane Collaboration, 2011. Available from https://www.cochrane-handbook.org

26. Netwerk Traumarevalidatie VUmc [http://traumarevalidatie.nl/].

27. Lamers L, Stalmeier P, McDonnell J, Krabbe P, van Busschbach J. Kwaliteit van leven meten in economische evaluaties: het Nederlandse EQ-5D-tarief. Ned Tijdschr Geneeskd. 2005;149:1574-8.

28. Hudak PL, Amadio PC, Bombardier C. Development of an upper extremity outcome measure: the DASH (disabilities of the arm, shoulder and hand) [corrected]. The upper extremity collaborative group (UECG). Am J Ind Med. 1996:29:602-8.
29. Veehof MM, Sleegers EJ, van Veldhoven $\mathrm{NH}$, Schuurman $\mathrm{AH}$, van Meeteren NL. Psychometric qualities of the Dutch language version of the disabilities of the arm, shoulder, and hand questionnaire (DASH-DLV). J Hand Ther. 2002;15:347-54.

30. Binkley JM, Stratford PW, Lott SA, Riddle DL. The Lower Extremity Functional Scale (LEFS): scale development, measurement properties, and clinical application. North American Orthopaedic rehabilitation research network. Phys Ther. 1999;79:371-83.

31. Hoogeboom TJ, de Bie RA, den Broeder AA, van den Ende $\mathrm{CH}$. The Dutch lower extremity functional scale was highly reliable, valid and responsive in individuals with hip/knee osteoarthritis: a validation study. BMC Musculoskelet Disord. 2012;13:117.

32. Roland M, Morris R. A study of the natural history of back pain. Part I: development of a reliable and sensitive measure of disability in low-back pain. Spine (Phila Pa 1976). 1983:8:141-4.

33. Brouwer S, Kuijer W, Dijkstra PU, Goeken LN, Groothoff JW, Geertzen JH. Reliability and stability of the Roland Morris disability questionnaire: intra class correlation and limits of agreement. Disabil Rehabil. 2004;26:162-5.

34. Kempen GI, Miedema I, Ormel J, Molenaar W. The assessment of disability with the Groningen Activity Restriction Scale. Conceptual framework and psychometric properties. Soc Sci Med. 1996;43:1601-10.

35. Jansen L, Steultjens MP, Holtslag HR, Kwakkel G, Dekker J. Psychometric properties of questionnaires evaluating health-related quality of life and functional status in polytrauma patients with lower extremity injury. J Trauma Manag Outcomes. 2010;4:7.

36. Von KM, Jensen MP, Karoly P. Assessing global pain severity by self-report in clinical and health services research. Spine (Phila Pa 1976). 2000;25:3140-51.

37. Chatman AB, Hyams SP, Neel JM, Binkley JM, Stratford PW, Schomberg A, Stabler M. The patient-specific functional scale: measurement properties in patients with knee dysfunction. Phys Ther. 1997;77:820-9.

38. Beurskens AJ, de Vet HC, Koke AJ, Lindeman E, van der Heijden GJ, Regtop W, Knipschild PG. A patient-specific approach for measuring functional status in low back pain. J Manip Physiol Ther. 1999:22:144-8.

39. Kamper SJ, Ostelo RW, Knol DL, Maher CG, de Vet HC, Hancock MJ. Global perceived effect scales provided reliable assessments of health transition in people with musculoskeletal disorders, but ratings are strongly influenced by current status. J Clin Epidemiol. 2010;63:760-6.

40. Azur MJ, Stuart EA, Frangakis C, Leaf PJ. Multiple imputation by chained equations: what is it and how does it work? Int J Methods Psychiatr Res. 2011;20:40-9.

41. White IR, Royston P, Wood AM. Multiple imputation using chained equations: issues and guidance for practice. Stat Med. 2011;30:377-99.

42. Twisk JWR. Applied longitudinal data analysis for epidemiology. A practical guide. Cambridge: Cambridge University Press; 2013.

43. Soer R, Reneman MF, Speijer BL, Coppes MH, Vroomen PC. Clinimetric properties of the EuroQol-5D in patients with chronic low back pain. Spine J. 2012;12:1035-9.

44. Parker SL, Mendenhall SK, Shau DN, Adogwa O, Anderson WN, Devin CJ, McGirt MJ. Minimum clinically important difference in pain, disability, and quality of life after neural decompression and fusion for same-level recurrent lumbar stenosis: understanding clinical versus statistical significance. J Neurosurg Spine. 2012;16:471-8.

45. Holtslag HR, Post MW, Lindeman E, Van der Werken C. Long-term functional health status of severely injured patients. Injury. 2007;38:280-9.

46. World Health Organisation. International classification of functioning, disability and health (ICF). Geneva: WHO; 2001

47. de Boer MR, Waterlander WE, Kuijper LD, Steenhuis IH, Twisk JW. Testing for baseline differences in randomized controlled trials: an unhealthy research behavior that is hard to eradicate. Int J Behav Nutr Phys Act. 2015;12:4.

\section{Publisher's Note}

Springer Nature remains neutral with regard to jurisdictional claims in published maps and institutional affiliations. 